\title{
PACO Piaractus brachypomus Y GAMITANA Colossoma macropomum CRIADOS EN POLICULTIVO CON EL BUJURQUI-TUCUNARÉ, Chaetobranchus semifasciatus (CICHLIDAE)
}

\author{
Jimmy TAFUR GONZALES ${ }^{1,2}$, Fernando ALCÁNTARA BOCANEGRA ${ }^{1,2,3}$, Marina DEL ÁGUILA \\ PIZARRO $^{2,3}$, Rosana CUBAS GUERRA $^{2,3}$, Luis MORI-PINEDO ${ }^{2,3}$, Fred CHU-KOO ${ }^{1,3}$ \\ 1 Instituto de Investigaciones de la Amazonía Peruana - IIAP. Programa para el Uso y Conservación del Agua y sus Recursos - \\ AQUAREC \\ 2 Facultad de Ciencias Biológicas. Universidad Nacional de la Amazonía Peruana - UNAP. \\ 3 Escuela de Postgrado de la Universidad Nacional de la Amazonía Peruana - UNAP. Cátedra CONCYTEC. Docente de la Maestría \\ en Acuicultura.
}

\section{RESUMEN}

Se evaluó el desempeño productivo de bujurqui-tucunaré Chaetobranchus semifasciatus, paco Piaractus brachypomus y gamitana Colossoma macropomum criados bajo el sistema de policultivo y alimentados con una dieta extrusada de $25 \%$ de proteína bruta durante 160 días. Un total de 900 peces (peso inicial de 6.5, 25.8 y 25.3 gramos para bujurqui-tucunaré, paco y gamitana, respectivamente) fueron asociados en tres policultivos (T1: bujurqui-tucunaré + paco, T2: bujurqui-tucunaré + gamitana y T3: bujurqui-tucunaré + paco + gamitana), distribuidos al azar dentro de nueve corrales de $100 \mathrm{~m}^{2}$, a razón de $1 \mathrm{pez} / \mathrm{m}^{2}$, densidad que se mantuvo hasta el final de estudio. La calidad del agua (transparencia, dióxido de carbono, alcalinidad, dureza, amonio, nitritos, temperatura, oxígeno disuelto y $\mathrm{pH}$ ) fue monitoreada periódicamente. Los tres policultivos produjeron interesantes tasas de crecimiento específico $(1.93,1.75$ y 1.75$)$ y pesos promedio finales $(153.5,450.4$ y $434 \mathrm{~g})$ en bujurquitucunaré, paco y gamitana, respectivamente, aunque en términos generales no influyeron significativamente $(\mathrm{P}>0.05)$ en un mejor desempeño productivo o en la conversión alimenticia de las especies estudiadas. Esta es la primera experiencia de cultivo publicada del bujurqui-tucunaré, cuyos resultados serán importantes para la piscicultura familiar en comunidades rurales e indígenas de la Amazonía, puesto que el uso de este cíclido planctófago nativo es una alternativa viable a la ilegal introducción de variedades de tilapia en selva baja.

PALABRAS CLAVE: Policultivo, rendimiento productivo, bujurqui-tucunaré, gamitana, paco.

\section{RED-BELLIED PACU Piaractus brachypomus AND BLACK-FINNED PACU Colossoma macropomum RAISED IN POLYCULTURE WITH BUJURQUI-TUCUNARÉ, Chaetobranchus semifasciatus (CICHLIDAE)}

\begin{abstract}
Yield performance of bujurqui-tucunaré Chaetobranchus semifasciatus, Red-bellied Pacu Piaractus brachypomus and Black-finned Pacu Colossoma macropomum raised under polyculture conditions and fed a $25 \%$ crude-protein extruded diet during 160 days was assessed. A total of 900 fish (initial weight of 6.5, 25.8 y $25.3 \mathrm{~g}$ for bujurquitucunaré, red-bellied and black-finned pacu, respectively) grouped in three polyculture systems (T1: bujurquitucunaré + red-bellied pacu, T2: bujurqui-tucunaré + black-finned pacu and T3: bujurqui-tucunaré + red-bellied pacu + black-finned pacu) were randomly placed into nine $100 \mathrm{~m}^{2}$ pens, following a stocking density of $1 \mathrm{fish} / \mathrm{m}^{2}$, which was maintained until the end of the experiment. Water quality (transparency, carbon dioxide, alkalinity, hardness, ammonia, nitrite, temperature, dissolved oxygen and $\mathrm{pH}$ ) was periodically monitored. All polycultures yielded interesting specific growth rates $(1.93,1.75$, and 1.75) and mean final weight $(153.5,450.4$, and $434 \mathrm{~g})$, in bujurqui-tucunaré, red-bellied pacu, and black-finned pacu, respectively; however, in general terms they did not significantly influenced $(\mathrm{P}>0.05)$ on a better yield performance or feed conversion rate of the fish species evaluated. The current study is the first experience accurately published on bujurqui-tucunare aquaculture whose outcomes will be important for family-based fish culture in rural and indigenous villages of the Peruvian Amazon, since using this native planctivore cichlid becomes in a viable alternative to the illegal introduction of several types of tilapia in lowland Amazonia.

KEYWORDS: Polyculture, yield performance, bujurqui-tucunaré, red-bellied pacu, and black-finned pacu.
\end{abstract}




\section{INTRODUCCIÓN}

La piscicultura es una actividad productiva importante y necesaria para asegurar en calidad y cantidad, el suministro de pescado para consumo humano directo en la región amazónica peruana (Chu-Koo \& Alcántara, 2007). Según las estadísticas del Ministerio de la Producción, la cosecha de especies amazónicas provenientes de acuicultura se incrementó paulatinamente de 320 a $700 \mathrm{TM}$ en los últimos cinco años (PRODUCE, 2009) y se estima que esta tendencia se mantenga en el próximo lustro. Paralelamente, la demanda de pescado por las poblaciones urbanas, rurales e indígenas de la Amazonía también se acrecentó, debido al rápido crecimiento poblacional, la escasez de pescado por efectos de la sobrepesca (García et al., 2009) y la contaminación de los ecosistemas acuáticos amazónicos. En este escenario, se hace necesario expandir la piscicultura de especies nativas como una medida exitosa de mitigar la escasez de pescado y generar renta en la Amazonía.

Las ventajas de desarrollar la piscicultura con especies nativas van desde la mejor adaptación de estos peces al clima y a la calidad del agua de la región, hasta el hábito tradicional de consumo de la población (Atencio-García, 2001). Otra razón que explica el alto potencial de crecimiento de la piscicultura amazónica está basada en la identificación de nuevas especies para el cultivo.

Entre las especies oriundas del Perú, el paco (Piaractus brachypomus) y la gamitana (Colossoma macropomum) son las más importantes y cultivadas (Chu-Koo \& Alcántara, 2007; Lochmann et al., 2009). Ambas, son de hábitos omnívoros con tendencia a la frugivoría (Piedade et al., 2006), nativas de la Amazonía (Saint-Paul, 1986), que han sido priorizadas para fines piscícolas en los países de la cuenca amazónica (Saint-Paul, 1984, 1985; Fernández et al., 2004), debido a su baja exigencia nutricional (proteína dietaria entre 18 a 32\% con alta asimilación de proteínas y lípidos de origen vegetal), rusticidad, buen crecimiento y rápida adaptación a varios tipos de alimentos y condiciones de cultivo (Deza et al., 2002; Rebaza et al., 2002; Ayllón \& Payahua, 2003; MesaGranda \& Botero-Aguirre, 2007; Gutiérrez et al., 2007), por lo que se recomiendan para la práctica de policultivo (Urbano et al., 2009).

Por otro lado, el cíclido Chaetobranchus semifasciatus, denominado en el IIAP como bujurquitucunaré; es un pez amazónico endémico de la cuenca del Yavarí y constituye desde el 2005 un registro nuevo para el país (Sánchez, 2005). Puede alcanzar 30 $\mathrm{cm}$ de longitud y pesar hasta $500 \mathrm{~g}$ y representa una especie con alto potencial para cultivos extensivos o de seguridad alimentaria por tratarse de una especie planctófaga, es decir, un organismo con bajo o nulo requerimiento de alimento balanceado (Sánchez, 2005; Ismiño et al., 2006). Es justamente esta característica que lo convierte en una especie promisoria para su utilización en condiciones de policultivo junto a especies de diferente hábitos alimenticios, mayor porte y valor económico, las mismas que por su calidad y demanda serían destinadas para la comercialización al público consumidor, siendo el cíclido destinado para el consumo directo de los piscicultores y/o los trabajadores de las fincas.

El policultivo es el cultivo simultáneo de dos o más especies de peces, que posean diferentes características y hábitos alimenticios, de manera que aprovechen más eficientemente los diferentes estratos y recursos alimentarios del estanque (FONDEPES, 2007).

El objetivo del presente estudio fue evaluar el crecimiento y los índices zootécnicos del bujurquitucunaré, el paco y la gamitana, criados bajo el sistema de policultivo.

\section{MATERIALES Y MÉTODOS}

\section{LUGAR DE ESTUDIO}

El estudio se ejecutó entre los meses de mayo a octubre de 2007 en el Centro de Investigaciones Quistococha (CIQ) del Instituto de Investigaciones de la Amazonía Peruana (IIAP), que se encuentra localizado en el Km. 4.5 de la Carretera Iquitos - Nauta, distrito de San Juan Bautista, provincia de Maynas, departamento de Loreto.

\section{PECES Y UNIDADES EXPERIMENTALES}

Se utilizó un total de 900 peces provenientes de los estanques de alevinaje del CIQ (60 bujurqui-tucunaré, 420 pacos y 420 gamitanas). Los pesos iniciales de los peces empleados en el estudio fueron de $6.5,25.8 \mathrm{y}$ 25.3 gramos para bujurqui-tucunaré, paco y gamitana, respectivamente.

\section{DISEÑO EXPERIMENTAL}

Se utilizaron tres policultivos como tratamientos experimentales $(\mathrm{T} 1=$ bujurqui-tucunaré + paco, $\mathrm{T} 2=$ bujurqui-tucunaré + gamitana y $\mathrm{T} 3=$ bujurquitucunaré + paco + gamitana). Los tratamientos fueron asignados aleatoriamente por triplicado en nueve corrales de $100 \mathrm{~m}^{2}(10 \mathrm{~m} \times 10 \mathrm{~m})$ construidos dentro de un estanque de $1000 \mathrm{~m}^{2}$. La densidad de cultivo fue 1 $\mathrm{pez} / \mathrm{m}^{2}$, siguiendo la distribución mostrada en la Tabla 1. 


\section{ALIMENTACIÓN DE LOS PECES}

Los peces fueron alimentados con una dieta extrusada con un tenor de $25 \%$ de proteína bruta y $2600 \mathrm{Kcal} / \mathrm{Kg}$ de energía digestible formulada y fabricada en la Planta de Producción de Alimentos Balanceados del IIAP. La tasa de alimentación empleada fue del $5 \%$ de la biomasa total de cada unidad experimental para el primer mes y $3 \%$ desde el segundo mes hasta el final del cultivo (160 días), con una frecuencia de alimentación de tres veces al día (08:00, 12:00 y 16:00 h) los siete días de la semana. Quincenalmente se evaluó el peso del $25 \%$ de la población de cada unidad experimental para evaluar el crecimiento y reajustar las raciones para las dos semanas subsecuentes.

\section{ÍNDICES ZOOTÉCNICOS}

Los índices zootécnicos evaluados fueron:

a) Índice de Conversión Alimenticia Aparente $(I C A A)=$ Total de alimento suministrado $/$ Total de biomasa ganada

b) Ganancia de Peso Diario $(G P D)=$ Ganancia de peso promedio / Duración del experimento

c) Tasa de Crecimiento Específico (TCE) = ln Peso promedio final - $\ln$ Peso promedio inicial / Tiempo * 100 d) Factor de Condición $(K)=$ Peso promedio final / Longitud promedio final ${ }^{3} * 100$

e) Sobrevivencia $(S)=$ Número de peces vivos al final / Número de peces vivos al inicio * 100

\section{CALIDAD DEL AGUA}

Se realizaron monitoreos diarios (08:00 y 16:00 h) de los niveles de temperatura del agua, oxígeno disuelto y $\mathrm{pH}$ con un medidor multiparámetros YSI MPS 556® (YSI Instrument Co., Ohio, USA) y monitoreos quincenales de amonio, nitritos, dureza, alcalinidad y $\mathrm{CO}_{2}$ con un Kit de Aguas Dulces AQ-2 (LaMotte Co., Maryland, USA). La transparencia del agua se midió con un disco Secchi.

\section{ANÁLISIS DE LOS DATOS}

Los datos fueron analizados utilizando el programa estadístico JMP IN versión 4.0.4, a través de análisis de varianza simple (alpha $=0.05)$, cuidando que se cumplan previamente todos los supuestos del ANOVA.

Tabla 1. Esquema de asociación de los ejemplares de bujurqui-tucunaré (Chaetobranchus semifasciatus), paco (Piaractus brachypomus) y gamitana (Colossoma macropomum) criados en policultivo durante 160 días en el Centro de Investigaciones Quistococha del IIAP.

\begin{tabular}{l|c|c|c}
\hline \multirow{2}{*}{ ESPECIE } & \multicolumn{3}{|c}{ TRATAMIENTOS (POLICULTIVOS) } \\
\cline { 2 - 4 } & $\begin{array}{c}\text { T1 } \\
\text { BUJ + PAC }\end{array}$ & $\begin{array}{c}\text { T2 } \\
\text { BUJ + GAM }\end{array}$ & $\begin{array}{c}\text { T3 } \\
\text { BUJ + PACO + GAM }\end{array}$ \\
\hline Bujurqui - tucunaré & 5 ind. & 5 ind. & 10 ind. \\
Paco & 95 ind. & --- & 45 ind. \\
Gamitana & --- & 95 ind. & 45 ind. \\
\hline
\end{tabular}




\section{RESULTADOS}

\section{CALIDADDEAGUA}

Los valores limnológicos registrados en las unidades de cultivo durante la ejecución del estudio fueron los siguientes: $29.7 \pm 0.9^{\circ} \mathrm{C}$ de temperatura del agua $5.8 \pm$ $0.7 \mathrm{mg} / \mathrm{L}$ de oxígeno disuelto, $6.2 \pm 0.2 \mathrm{de} \mathrm{pH}, 4.9 \pm$ $1.2 \mathrm{mg} / \mathrm{L}$ de $\mathrm{CO}_{2}, 17.1 \pm 1.1 \mathrm{mg} / \mathrm{L}$ de alcalinidad y 21.7 $\pm 1.8 \mathrm{mg} / \mathrm{L}$ de dureza total. La transparencia fue de $32.0 \pm 2.3 \mathrm{~cm}$, el amonio siempre se mantuvo por debajo de $0.2 \mathrm{mg} / \mathrm{L}$ y los nitritos estuvieron siempre inferiores a $0.05 \mathrm{mg} / \mathrm{L}$. En líneas generales, todos los parámetros evaluados se mantuvieron dentro de los rangos considerados normales para el cultivo de especies amazónicas (Argumedo \& Rojas, 2000).

\section{CRECIMIENTO DE LOS PECES}

Según los análisis de varianza realizados, ningún policultivo influyó decisivamente en el rendimiento productivo o en la utilización del alimento de las especies en estudio $(\mathrm{P}>0.05)$. En tal sentido, el peso promedio final, la ganancia de peso, la ganancia de peso diario, la tasa de crecimiento específico y el factor de condición de las tres especies de peces no mostraron diferencias significativas al término del estudio ( $\mathrm{P}>0.05$, Tablas 2 y 3$)$.

Tabla 2. Índices zootécnicos (promedio \pm D.S.) de bujurqui-tucunaré Chaetobranchus semifasciatus criado en policultivo con paco (Piaractus brachypomus) y gamitana (Colossoma macropomum) durante 160 días.

\begin{tabular}{l|c|c|c}
\hline Í́nDICES Z00TÉCNICOS & $\begin{array}{c}\text { T1 } \\
\text { (BUJ + PAC) }\end{array}$ & $\begin{array}{c}\text { T2 } \\
\text { (BUJ + GAM) }\end{array}$ & $\begin{array}{c}\text { T3 } \\
\text { (BUJ + PACO + GAM) }\end{array}$ \\
\hline Peso Promedio Inicial (g) & $6.6 \pm 0.1$ & $6.4 \pm 0.5$ & $6.3 \pm 0.3$ \\
\hline Peso Promedio Final (g) & $153.7 \pm 16.6$ & $164.0 \pm 19.3$ & $142.9 \pm 15.1$ \\
Ganancia de Peso (g) & $147.1 \pm 16.6$ & $157.6 \pm 18.9$ & $136.5 \pm 15.0$ \\
Ganancia de Peso Diario (g) & $0.9 \pm 0.1$ & $1.0 \pm 0.1$ & $0.8 \pm 0.1$ \\
Factor de Condición & $1.8 \pm 0.2$ & $1.9 \pm 0.03$ & $1.9 \pm 0.02$ \\
\hline Tasa de Crecim. Específico & $1.9 \pm 0.1$ & $2.0 \pm 0.1$ & $1.9 \pm 0.1$ \\
\hline Producción (kg/Ha/año) & 153.7 & 164.0 & 285.8 \\
\hline Sobrevivencia (\%) & 100 & 100 & 100 \\
\hline
\end{tabular}




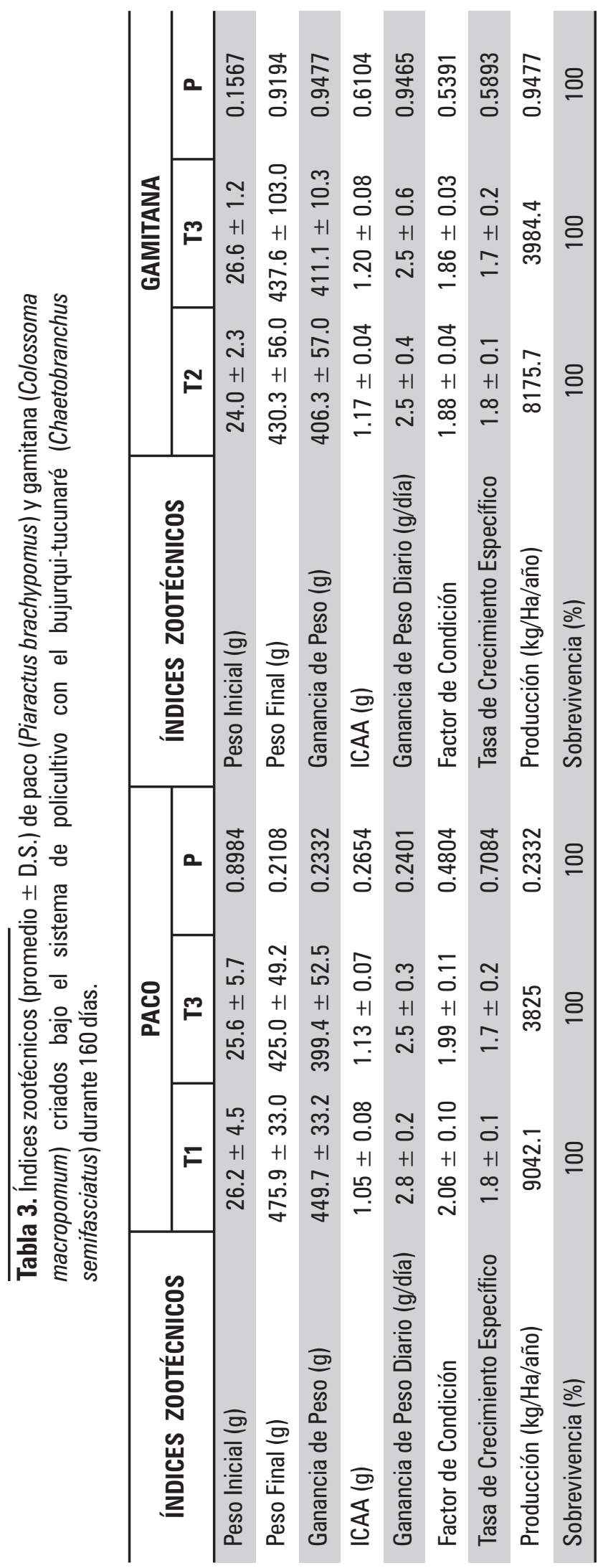




\section{DISCUSIÓN}

\section{CRECIMIENTO DE LOS PECES}

Los tres policultivos evaluados, produjeron interesantes tasas de crecimiento específico (1.93, 1.75 y 1.75$)$ y pesos promedio finales $(153.5,450.4$ y $434 \mathrm{~g})$ en bujurqui-tucunaré, paco y gamitana, respectivamente. Posiblemente estos resultados no sean muy impactantes en el caso del paco y la gamitana, cuya fácil adaptación a diversas condiciones de cultivo, fuentes alimenticias y buen rendimiento productivo son ampliamente conocidas (Ponte et al., 1992; Ascón et al., 2003); sin embargo, en el caso del bujurqui-tucunaré, una especie totalmente nueva para la piscicultura, el presente estudio comprueba la factibilidad técnica de su crianza en asociación con peces omnívoros, obteniéndose en el lapso de poco más de cinco meses de cultivo, ejemplares que promediaban los 153.5 gramos de peso a partir de alevinos de apenas 6.5 gramos, un crecimiento inmensamente superior a los resultados reportados por Mori (1993) en el bujurqui vaso Chaetobranchus flavescens, una especie amazónica cercana, criada durante nueve meses en el Centro Experimental del IIAP en Jenaro Herrera, la que solo alcanzó como máximo $12.9 \mathrm{~g}$ de peso promedio final con alimento balanceado.

Si analizamos el crecimiento de las tres especies en términos de productividad por hectárea de espejo de agua, incluyendo también el tiempo que tomaría cosechar los peces, vaciar, desinfectar, fertilizar y volver a llenar el estanque para una segunda siembra, se llega a la conclusión básica de que se podría realizar dos cosechas por año y alcanzar niveles de producción de pescado de $9.2,8.3$ y 8.0 ton/ha/año para los tratamientos T1, T2 y T3, respectivamente.

Nuestros resultados confirman el buen desempeño productivo del paco y la gamitana en condiciones de policultivo, algo que también fue reportado por Ascón et al. (2003), quienes al ejecutar un policultivo de gamitana y boquichico (pesos iniciales de $20.2 \mathrm{~g}$ ), obtuvieron pesos finales de $674.8,672.5$ y $653.33 \mathrm{~g}$, en gamitanas alimentadas con dietas de contenido proteico de 20, 22 y $25 \%$, respectivamente, en 12 meses de cultivo. Por su parte, Ponte et al. (1992) en un policultivo de gamitana, carpa común y tilapia nilótica asociada a patos; obtuvo pesos medios finales de $633.3,430$ y 246 gramos en gamitana, carpa y tilapia, respectivamente a partir de alevinos de 12,7 y 23 gramos en 6 meses de cultivo. Varios autores han reportado que el paco y la gamitana alcanzan normalmente entre los 300 y $600 \mathrm{~g}$ entre los 4 a 6 meses de cultivo (Teichert-Coddington et al., 1996; Campos-Baca \& Kohler, 2005; Lochmann et al., 2009). En ese sentido el peso promedio alcanzado en la presente investigación se encuentra dentro de lo esperado en el cultivo de estas especies.

Los excelentes índices de conversión alimenticia aparente registrados en paco y gamitana (1.09 y 1.18, respectivamente), indican la fácil y rápida adaptación de estas dos especies al tipo de alimento. Asimismo, es un indicador de la alta calidad de la dieta utilizada, aunque no debemos descartar, la influencia de otras fuentes alimenticias autóctonas como el zooplancton del propio estanque, puesto que la gamitana y el paco, tienen capacidad para aprovechar estos organismos de alto valor nutricional. Los ICAA reportados en el presente estudio son similares a los reportados por Gutiérrez et al., 2007; Rebaza et al., 2008), e incluso más eficientes que los registrados por otros autores en estudios de alevinaje y engorde de paco y gamitana (Gomes et al., 2006; Chagas et al., 2007; Soberón et al., 2007; Casanova \& Chu-Koo, 2008; Lochmann et al., 2009). Por otro lado, no se registró mortalidad durante el periodo de estudio, lo que demuestra la rusticidad de las especies utilizadas y que en el caso de gamitana y paco fueron reportados en la literatura (Rebaza et al., 2002; Chagas \& Val, 2003; Chuquipiondo \& Galdós, 2005).

Considerando el peso promedio final alcanzado por el bujurqui-tucunaré, se podría inferir que un monocultivo de esta especie podría resultar en dos cosechas por año, con rendimientos de hasta 3070 $\mathrm{kg} / \mathrm{ha} / \mathrm{año} \mathrm{(incrementando} \mathrm{la} \mathrm{actual} \mathrm{densidad} \mathrm{de} 0.05 \mathrm{a}$ 1 bujurqui-tucunaré $/ \mathrm{m}^{2}$ ), con la ventaja de no utilizar alimentos balanceados y manejando la alimentación de los peces solo con prácticas periódicas de fertilización de las aguas del estanque con estiércol de aves, cerdos y/o hierba seca para mantener una producción constante de plancton en el ambiente acuático. Estudios adicionales deberían ser realizados para mejorar, validar o en todo caso desechar esta hipótesis puesto que el mantenimiento de la productividad planctónica es un punto crítico para el sustento de una creciente biomasa de peces. Adicionalmente, existen evidencias de que ciertas especies crecen mejor cuando están asociadas a otros peces, de los cuales aprovechan las sobras de alimentos y las excretas para su alimentación (Urbano et al., 2009; CCI, 2010). Uno de estos estudios fue realizado en Venezuela y reportó que el coporo (Prochilodus mariae) criado en policultivo a la densidad de $1 \mathrm{pez} / \mathrm{m}^{2}$ junto a la gamitana, alcanza hasta $242 \mathrm{~g}$ de peso en seis meses, mientras que en monocultivo solo llega a pesar $102 \mathrm{~g}$ (Urbano et al., 2009).

El presente trabajo abre nuevas posibilidades para la práctica de la piscicultura familiar y/o de seguridad alimentaria en comunidades rurales e indígenas de la Amazonía, puesto que el uso de este cíclido 
planctófago nativo se convierte en una alternativa técnica y ecológicamente viable a la introducción de las variedades modificadas de tilapia africana, un cíclido exótico conocido por su alta capacidad de colonizar nuevos ambientes y desplazar a las especies nativas. Cabe destacar que el presente estudio es la primera experiencia de cultivo debidamente reportada para el bujurqui-tucunaré.

\section{CONCLUSIONES}

Los policultivos evaluados produjeron un crecimiento homogéneo, ninguna mortalidad e interesantes tasas de crecimiento específico y conversión alimenticia aparente en las especies estudiadas, aunque en términos generales no influyeron significativamente en un mejor desempeño productivo o conversión alimenticia de las especies estudiadas. El presente estudio es la primera experiencia de cultivo publicada del bujurqui-tucunaré, cuyos resultados abren la posibilidad del uso de este cíclido planctófago nativo como una alternativa viable a la introducción de variedades modificadas de tilapia africana en selva baja. Por el nivel de crecimiento y el tipo de requerimiento alimenticio, el mayor potencial de uso de esta especie estaría enfocado a la práctica de la piscicultura familiar o de seguridad alimentaria en comunidades rurales e indígenas de la Amazonía.

\section{BIBLIOGRAFÍA CITADA}

Argumedo, T. E. G.; Rojas, D. H. M. 2000. Manual de piscicultura con especies nativas. Asociación de Acuicultores del Caquetá - ACUICA. Bogotá, Colombia. 151p.

Ascón, G.; Guerra, H.; Iberico, L. 2003. Policultivo de gamitana, Colossoma macropomum más boquichico, Prochilodus nigricans durante 24 meses, en tres fases consecutivas de cultivo. Reporte Técnico. Programa de Ecosistemas Acuáticos, IIAP. 16p.

Atencio-García, V. 2001. Producción de alevines de especies nativas. MVZ Córdova, 6(1).9-14

Ayllón, Z.; Payahua, J. 2003. Uso de la harina de pijuayo (Bactris gasipaes H.B.K. 1815), en la alimentación del paco (Piaractus brachypomus, Cuvier 1818), criado en ambientes controlados. Tesis para optar el título de Biólogo. Universidad Nacional de la Amazonía Peruana. Iquitos-Perú. $63 \mathrm{p}$.

Campos-Baca, L.; Kohler, C. 2005. Aquaculture of Colossoma macropomum and related species in Latin America. American Fisheries Society Symposium, 46:451-561.
Casanova F. R.; Chu-Koo, F. 2008. Evaluación del polvillo de malta de cebada, Hordeum vulgare, como insumo alimenticio para gamitana (Colossoma macropomum). Folia Amazónica, 17(1-2):15-22.

Chagas, E.C.; Val, A.L. 2003. Efeito da vitamina C no ganho de peso e em parámetros hematológicos de tambaqui. Pesquisa Agropecuária Brasileira, 38(3):397-402.

Chagas, E. C.; Gomes, L. C.; Martins J. H.; Roubach, R. 2007. Produtividade de tambaqui criado em tanque-rede com diferentes taxas de alimentação. Ciencia Rural, 37(4):1109-1115.

ão. Ciencia Rural, 37(4):1109-1115.

Chu-Koo, F. W.; Alcántara, F. 2007. De la selva su acuicultura. Sobre los avances en acuicultura en la Amazonía peruana y las oportunidades de inversión. Perú Económico, 30(1):11-12.

Chuquipiondo, J. M. L.; Galdos, R. A. P. 2005. Influencia de la harina de plátano, Musa paradisiaca $\mathrm{L}$. en el crecimiento de alevinos de gamitana Colossoma macropomum (Cuvier, 1818). Tesis para optar el Título de Biólogo. Universidad Nacional de la Amazonía Peruana. Iquitos, Perú. 78p.

Deza, S.; Quiroz, S.; Rebaza, M.; Rebaza, C. 2002. Efecto de la densidad de siembra en el crecimiento de Piaractus brachypomus (Cuvier, 1818) "paco" en estanques seminaturales de Pucallpa. Folia Amazónica, 13 (1-2):49-64.

Fernandes, J. B. K.; Lochmann, R.; Alcántara, F. B. 2004. Apparent digestible energy and nutrient digestibility coefficients of diet ingredients for pacu Piaractus brachypomus. Journal of the World Aquaculture Society, 35:237-244.

FONDEPES. 2007. Guía Técnica: Policultivo de peces tropicales en la Amazonía. 2da. Edición. Lima, Perú. 37p.

García, A.; Tello, S.; Vargas, G.; Duponchelle, F. 2009. Patterns of commercial fish landings in the Loreto region (Peruvian Amazon) between 1984 and 2006. Fish Physiology and Biochemistry, 35:5367.

Gomes, L. C.; Chagas, E. C.; Martins-Junior, H.; Roubach, R.; Ono, E. A.; Lourenço, J. N. P. 2006. Cage culture of tambaquí (Colossoma macropomum) in a central Amazon floodplain lake. Aquaculture, 253(1-4):374-384.

Gutiérrez, F. W.; Quispe, M.; Valenzuela, L.; Contreras, G.; Zaldívar, J. 2007. Utilización de la proteína dietaria por alevinos de gamitana Colossoma macropomum Cuvier 1818, alimentados con dietas isocalóricas. Folia Amazónica, 16(1-2): 47-53. 
Ismiño, R.; Sánchez, H.; García, A.; Chávez, C.; ChuKoo F. W. 2006. Análisis del contenido estomacal del bujurqui - tucunaré Chaetobranchus semifasciatus cultivados en estanques piscícolas de la Amazonía Peruana. 2do. Congreso Nacional de Acuicultura. La Molina, Lima.

Lochmann, R.; Chen, R.; Chu-Koo, F. W.; Camargo, W.; Kohler, C. C. 2009. Effects of carbohydraterich alternative feedstuffs on growth, survival, body composition, hematology, and non-specific immune response of Black Pacu, Colossoma macropomum, and Red Pacu, Piaractus brachypomus. Journal of the World Aquaculture Society, 40(1): 33-44.

Mesa-Granda, M. N.; Botero-Aguirre, M. C. 2007. La cachama blanca (Piaractus brachypomus), una especie potencial para el mejoramiento genético. Revista Colombiana de Ciencias Pecuarias, 20(1): 79-86.

Mori, P. L. A. 1993. Estudio de la crianza en cautiverio del bujurqui vaso Chaetobranchus flavescens (Heckel, 1840), (Pisces, Cichlidae) alimentado con dos tipos de dietas en Jenaro Herrera Provincia de Requena, Región Loreto. Tesis para Optar el Título de Biólogo. Universidad Nacional de la Amazonía Peruana - UNAP. Iquitos, Perú. $74 \mathrm{p}$.

Piedade, M. T. F.; Parolin, P.; Junk, W. G. 2006. Phenology, fruit production and seed dispersal of Astrocaryum jauari (Arecaceae) in Amazonian black water floodplains. International Journal of Tropical Biology, 54 (4):1171-1178.

Ponte, F.C.A.; Silva, J.; Carneiro, S.A.; Bezerra, A. 1992. Resultados de um ensaio de policultivo de tambaqui, Colossoma macropomum. Cuvier, 1818: carpa espelho, Cyprinus carpio L., 1785 vr. specularis, e macho da tilapia do Nilo, Oreochromis niloticus L., 1766, consorciado com marreco de pequin, Anas platyrinchus. Ciência Agronómica, 23(1-2): 93-102.

PRODUCE. 2009. Perú: Cosecha de recursos hidrobiológicos procedentes de la actividad de acuicultura por ámbito según especie 2000-2008. Anuario Estadístico 2008. Lima, Perú. p118.
Rebaza, C.; Villafana, E.; Rebaza, M.; Deza, S. 2002. Influencia de tres densidades de siembra en el crecimiento de Piaractus brachypomus. "paco" en segunda fase de alevinaje en estanques seminaturales. Folia Amazónica, 13 (1-2): 122 134.

Rebaza, C.; Valdivieso, M.; Rebaza, M.; Chu-Koo, F. 2008. Análisis económico del cultivo de gamitana Colossoma macropomum y paco Piaractus brachypomus usando una dieta extrusada comercial en Ucayali. Folia Amazónica, 17(12):7-14.

Saint-Paul, U. 1984. Ecological and physiological investigations on Colossoma macropomum, a new specie for fish culture in Amazonas. Memorias de la Asociación Latinoamericana de Acuicultura, 5(3):501-518.

Saint-Paul, U. 1985. The neotropical Serrasalmid Colossoma macropomum, a promising species for fish culture in Amazonia. Animal Research and Development, 22(2):7-31.

Saint-Paul, U. 1986. Potential for aquaculture of South American freshwater fishes: a review. Aquaculture, 54: 205-240

Sánchez, H. 2005. Presencia de Chaetobranchus semifasciatus Steindachner, 1875 (Cichlidae), en la cuenca del río Yavarí (Loreto-Perú). Folia Amazónica, 15(2):105-108.

Soberón, M. L. E.; Chu-Koo, F.; Alcántara, B. F. 2007. Parámetros hematológicos, crecimiento y composición corporal de juveniles de gamitana Colossoma macropomum (Cuvier, 1818) cultivados bajo tres densidades de siembra. Folia Amazónica, 16(1-2): 35-45.

Teichert-Coddington, D. R. 1996. Effect of stocking ratio on semi-intensive polyculture of Colossoma macropomum and Oreochromis niloticus in Honduras, Central America. Aquaculture, 143 (34): 291-302

Urbano, T.C.; Moreno, C.; Silva, A.; Santaimé, R. 2009. Engorde de coporo en lagunas de tierra, en el estado Delta Amacuro. Boletin INIA Divulga, 12(1):7-10. 

